\title{
High-resolution spectroscopic monitoring of SV Camelopardalis
}

\section{Orbits, absolute masses and radii of the components}

\author{
H. Lehmann ${ }^{1}$, A. Hempelmann ${ }^{2}$, and U. Wolter ${ }^{2}$ \\ 1 Thüringer Landessternwarte, 07778 Tautenburg, Germany \\ 2 Universität Hamburg, Hamburger Sternwarte, Gojenbergsweg 112, 21029 Hamburg, Germany \\ e-mail: ahempelmann@hs.uni-hamburg.de, uwolter@hs.uni-hamburg.de
}

Received 22 May 2002 / Accepted 27 June 2002

\begin{abstract}
This study is a first part of an analysis of newly obtained time series of high-resolution échelle spectra of the RS CVn star SV Cam. We investigate the radial velocity variations of the spectroscopic binary and derive an improved orbital solution for both components. The results allow us to estimate the masses of the primary to $1.1 M_{\odot}$ and of the secondary to $0.7 M_{\odot}$ and, from a previous analysis of the lightcurve of SV Cam, we derive the stellar radii of $1.18 R_{\odot}$ for the primary and of $0.76 R_{\odot}$ for the secondary. From a compilation of eclipse minimum times spanning a period of over 100 years we compute the orbital elements of a hypothetical third body belonging to the SV Cam system. We obtain a period of 52.3 years which is slightly longer than derived by authors before and a much higher eccentricity of at least 0.42 . From the mass function there follows a lower limit to the third body mass of $0.17 M_{\odot}$. The applied procedure also provides an optimized period of eclipses of 0.59307180 .
\end{abstract}

Key words. stars: binaries: spectroscopic - stars: binaries: eclipsing - stars: fundamental parameters stars: variables: general - stars: individual: SV Cam

\section{Introduction}

The RS CVn binary star SV Cam is one of the best studied magnetically active stars. Eclipsing light-curves have been observed for more than 100 years. Light-curve analysis as well as spectroscopic studies have yielded not only more or less precise stellar parameters but also indications of spots on the primary star. Our intention is to continue the monitoring of SV Cam's activity, mainly by high signal-to-noise spectroscopy. We started these observations at the 2-m telescope of the Tautenburg Observatory in 2000. While our main task is Doppler imaging and monitoring of star spots on the primary star of SV Cam the observations also result in radial velocities $(R V \mathrm{~s}$ hereinafter) with much higher precision than obtained in earlier studies. Our knowledge of the secondary star in particular is significantly improved.

Although the primary star of SV Cam is well studied, there are only a few direct observations of the secondary, among them a spectroscopic determination of its orbit by Pojmanski (1998) as well as several determinations of stellar parameters from eclipse analysis (e.g. Budding \& Zeilik 1987; Zeilik et al. 1988; Patkòs \& Hempelmann 1994). One of the most recent studies of the composite spectrum of SV Cam was done by Özeren et al. (2001). The authors conclude that most of

Send offprint requests to: $\mathrm{H}$. Lehmann, e-mail: lehm@tls-tautenburg.de the chromospheric activity features (excess emission components in the Balmer lines) originate from the cool secondary component. This conclusion also confirms earlier results by Hempelmann et al. (1997) based on the ROSAT X-ray fluxes and on optical spectroscopy. Our new spectra of SV Cam clearly show spectral lines of the secondary star which allows a redetermination of its orbit and of the mass ratio based on a large number of high quality data. This will be done in Sects. 4 and 6.

Another problem which remains somewhat open is a possible variability of the orbital period. Many authors tried to fit the $\mathrm{O}-\mathrm{C}$ diagram of the times of eclipse minima by a sinusoidal oscillation and determined a period in the range of 40 to 80 years (Van Worden 1957; Frieboes-Conde \& Herczeg 1973; Hilditch et al. 1979; Cellino et al. 1985; Albayrak et al. $2001=$ ADD hereafter). Such regular behaviour can be explained either by rotation of the apsidal line or by a third body. Frieboes-Conde \& Herczeg (1973) could exclude the first possibility because the orbital eccentricity required for apsidal motion should be very high, which is not observed.

One possible cause for long-term variability of the orbital period may be stellar activity: either by magnetically induced mass exchange (Hempelmann \& Patkòs 1991) or by the stellar dynamo (Lanza \& Rodonò 1999). The observed period lengths on the order of half a century or more are in strong contrast to an expected activity cycle on the order of ten years 
Table 1. Total number of $R V \mathrm{~s} N$, number of $R V \mathrm{~s}$ used for the determination of the primary orbit $N_{\text {orb }}$ ( $R V \mathrm{~s}$ near to primary eclipse were excluded), and epochs of observation. The applied weights and the individual $\gamma$-velocities are taken from orbital solution $A_{2}$ (see Table 3). Sources are: (1) Hiltner (1953), (2) Rainger et al. (1991), (3) Pojmanski (1998), (4) this paper.

\begin{tabular}{cccccc}
\hline \hline source & $N$ & $N_{\text {orb }}$ & JD 2 400 000+ & weight & $\gamma\left[\mathrm{km} \mathrm{s}^{-1}\right]$ \\
\hline$(1)$ & 46 & 40 & $32244-32295$ & 3 & $-14.0 \pm 1.4$ \\
$(2)$ & 24 & 22 & $47107-47202$ & 2 & $-14.1 \pm 1.5$ \\
$(3)$ & 34 & 30 & $49350-49354$ & 5 & $-13.8 \pm 1.1$ \\
$(4)$ & 151 & 128 & $51971-52059$ & 100 & $-13.9 \pm 0.1$ \\
\hline
\end{tabular}

Table 2. Spectral lines which were used to investigate the contribution by the secondary. Contributions to blends are given in brackets, corresponding weights in parentheses.

\begin{tabular}{ll}
\hline \hline Fe I & 5397.1315662 .525 \\
& $5615.577[5615.308(126), 5615.652(452)]$ \\
& $5658.725[5658.542(95), 5658.670(74), 5658.826(214)]$ \\
& $6137.114[6136.620(137), 6136.999(64), 6137.696(129)]$ \\
$\mathrm{Ca} I$ & 6102.7226122 .2196162 .172 \\
& $6169.325[6169.055(85), 6169.559(98)]$ \\
\hline
\end{tabular}

(Busso et al. 1985), however. Hence, the third body hypothesis is the most attractive. We investigate the long-term variations on the basis of this hypothesis in Sect. 5.

\section{Observations and data reduction}

Spectra were obtained with the Coude échelle spectrograph of the Tautenburg 2-m telescope. They cover the wavelength range from 4700 to $7200 \AA$ with a resolution of about 40000 and a typical signal-to-noise ratio of 150 . In all, 151 spectra were taken within 13 nights during 2001 March to May with a single exposure time of $10 \mathrm{~min}$.

For the reduction of the échelle spectra we used standard MIDAS routines. Reduction included filtering of image distortions due to cosmic rays and hot pixels, flat fielding, optimum extraction of spectra, wavelength calibration by a Th-Ar lamp, wavelength correction by telluric absorption lines, and continuum normalization.

RVs were measured by cross-correlating the logarithmic rebinned spectra in the wavelength range from 4875 to $5550 \AA$. In this region the spectrum of SV Cam is almost free of telluric lines and of night sky emission. The $\mathrm{H}_{\beta}$ region was excluded because the Balmer lines show an extraordinary line profile variation which we will discuss in a following paper on line profile variations in general. In a first step one arbitrary spectrum served as template. Then all spectra were shifted according to the derived $R V \mathrm{~s}$. A new template was built from the average of shifted spectra and the cross-correlation was repeated with the new template. The resulting $R V \mathrm{~s}$ were shifted for the $R V$ of the template which was determined from several lines of $\mathrm{Ca} I$ and Fe I which we also used to determine the orbit of the secondary (Sect. 4 and Table 2). The heliocentric correction was applied to the $R V \mathrm{~s}$ as well as to the dates of mean exposure.

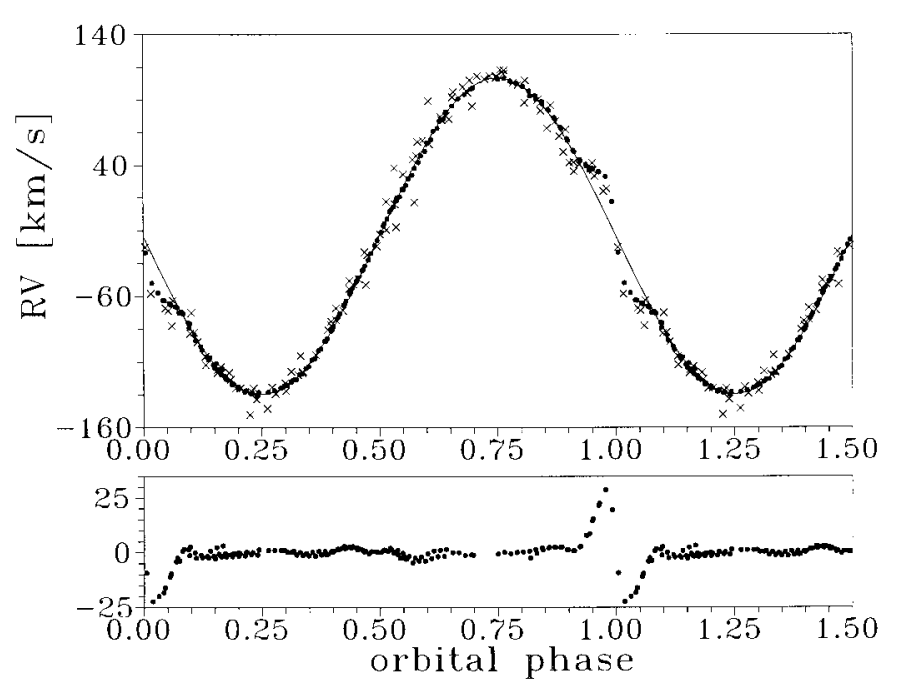

Fig. 1. Orbital solution $A_{2}$ (see Table 3) of SV Cam assuming a circular orbit. $R V \mathrm{~s}$ from TLS spectra are marked by dots, $R V \mathrm{~s}$ from literature by crosses. Residuals are shown below for the TLS data.

Beside our own measurements we used a compilation of $R V \mathrm{~s}$ taken from the literature. Table 1 lists the various data sources.

\section{Orbital solution}

Orbital elements were computed by the method of differential corrections (Schlesinger 1908). This procedure optimizes the orbital elements and the orbital period simultaneously. To compute the orbital solution we excluded all $R V \mathrm{~s}$ which show a larger deviation from the orbital curve due to transit effects during primary eclipse. Table 3 lists the results. They were derived assuming circular orbits (solutions $A$ ) as well as non-circular orbits (solutions $B$ ). Solutions $A_{1}$ and $B_{1}$ were determined from the data obtained at the Türinger Landesternwarte (TLS) alone. Here the resulting rms of the residuals of about $1.5 \mathrm{~km} \mathrm{~s}^{-1}$ does not reflect the error of measurement which is about $0.3 \mathrm{~km} \mathrm{~s}^{-1}$ but arises from temporary intrinsic variations (see bottom panel of Fig. 1).

Although our data are of much higher precision than the data from the literature, it is not possible to derive an orbital solution which is based only on our $R V \mathrm{~s}$ and which also fits the older data. The reason is that the time base of our observations is too small to obtain a sufficiently precise orbital period.

For computing a common orbital solution from all available data we had to weight the $R V$ s from different sources and to check for their individual $R V$ zero-points. First we calculated a common solution from the unweighted data. Then we determined the mean and the mean scatter of the residuals for each data set separately. The mean served as the individual $\gamma$-correction. The weight of each data set was defined by the inverse squared mean scatter of its residuals. This procedure was repeated until convergence was reached. From the weighted and corrected data we get the orbital elements listed in Table 3 as solutions $A_{2}$ and $B_{2}$. Table 1 lists the weights which were normalized to 100 for TLS $R V \mathrm{~S}$ and the individal $\gamma$-velocities obtained from solution $A_{2}$. It can be seen that 
Table 3. Orbital elements of SV Cam obtained for circular (solutions $A$ ) and non-circular (solutions $B$ ) orbits from TLS data (solutions 1) and from all data (solutions 2). $T$ refers to the epoch of primary eclipse which was calculated in the case of non-circular orbits from the time of periastron passage $T_{\mathrm{P}}$. Errors of the last digit are given in parenthesis. rms was computed from the weighted mean scatter of the residuals.

\begin{tabular}{|c|c|c|c|c|c|c|c|c|c|}
\hline & solution & $\begin{array}{l}P \\
\mathrm{~d}\end{array}$ & $\begin{array}{c}T \\
2452011+\end{array}$ & $\begin{array}{c}K_{1} \\
\mathrm{~km} \mathrm{~s}^{-1}\end{array}$ & $\begin{array}{c}\gamma \\
\mathrm{km} \mathrm{s}^{-1}\end{array}$ & $e$ & $\begin{array}{c}T_{\mathrm{P}} \\
2452011+\end{array}$ & $\begin{array}{c}\omega \\
\text { deg }\end{array}$ & $\begin{array}{c}\mathrm{rms} \\
\mathrm{km} \mathrm{s}^{-1}\end{array}$ \\
\hline \multirow[t]{2}{*}{$e=0$} & $A_{1}$ & $0.593084(3)$ & $0.4213(2)$ & $-120.4(2)$ & $-14.1(2)$ & - & - & - & 1.55 \\
\hline & $A_{2}$ & $0.5930720(4)$ & $0.4212(2)$ & $-120.5(2)$ & $-13.9(2)$ & - & - & - & 2.13 \\
\hline \multirow[t]{2}{*}{$e>0$} & $B_{1}$ & $0.593079(3)$ & $0.42(3)$ & $-120.4(2)$ & $-13.9(1)$ & $0.009(2)$ & $0.46(3)$ & $12(1)$ & 1.35 \\
\hline & $B_{2}$ & $0.5930720(4)$ & $0.42(3)$ & $-120.4(2)$ & $-13.8(1)$ & $0.010(2)$ & $0.47(3)$ & $12(1)$ & 1.83 \\
\hline
\end{tabular}

the $\gamma$-velocities are constant within the individual errors and that the applied $\gamma$-corrections should not effect the orbital solution. The influence of the applied weighting scheme will be discussed in Sect. 7.

\section{Orbit of the secondary}

Contributions from the secondary could be detected in the $\mathrm{Ca}$ I lines and in some of the Fe I lines. Because of the large amplitude of the orbital $R V$ variation $\left(\sim 120 \mathrm{~km} \mathrm{~s}^{-1}\right.$ for the primary and $\sim 190 \mathrm{~km} \mathrm{~s}^{-1}$ for the secondary) and the opposite sign of these variations most of the lines of the secondary are strongly blended by neighbouring lines of the primary, at least near the extrema of the orbital $R V$ curve. So we only used a few selected lines to investigate the secondary. Because of the large $v \sin i$ most of these lines consist of blends. Table 2 lists the selected lines and gives the single line contributions to each blend in brackets. The wavelength of the blends was computed by weighting the single contributions by their equivalent widths as given in the atlas of solar lines (Moore et al. 1966).

In a first step all spectra were superimposed into a twodimensional frame showing the entire time series in the $(R V$, orbital phase) plane. Phase was binned into 50 steps from 0 to 1 , spectra which overlap in phase were averaged. The result can be seen in the left panel of Fig. 2. Then we shifted all spectra according to the obtained orbital solution to the rest frame of the primary (Fig. 2, middle) and averaged all columns to one background frame which is dominated by the line profile of the primary and some small and smeared out contribution from the secondary. The subtraction of this background frame from the previous one shows the variation of the secondary lines much clearer (Fig. 2, right).

To obtain maximum signal-to-noise, $R V \mathrm{~s}$ of the secondary were measured row-by-row in the shifted and backgroundsubtracted phase plot as it is shown in the right panel of Fig. 2. Derived $R V \mathrm{~s}$ were corrected for the shift due to the orbital motion of the primary. Then we fitted the orbital solution obtained for the primary to the $R V \mathrm{~s}$ of the secondary where we allowed only for a variable half-amplitude $K_{2}$. Measurements close to the eclipses are very uncertain and were excluded. As result we get $K_{2}=-187 \pm 4 \mathrm{~km} \mathrm{~s}^{-1}$. Figure 3 shows the orbital solutions for both components.

\section{Photometric minima and the third body orbit}

In the following we want to compare our spectroscopically derived orbit with the times of primary minima in the light-curve
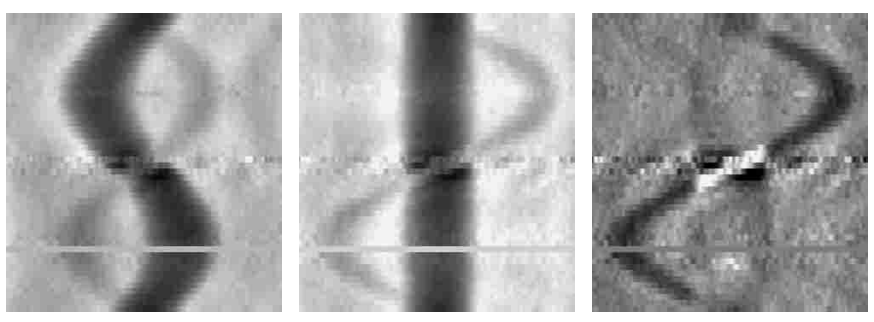

Fig. 2. Time series of spectra, superposition of all lines listed in Table 2. The vertical axis gives the orbital phase from -0.5 (bottom) to +0.5 (top), the horizontal axis spans a range in $R V$ of $\pm 400 \mathrm{~km} \mathrm{~s}^{-1}$. There is a horizontal strip with a lower population of spectra and enhanced noise at phase zero (primary conjunction) and a grey strip near to phase -0.3 without any phase coverage. From left to right: a) variation due to the orbital motions of the primary and the secondary, b) line profiles shifted to the rest frame of the primary, c) primary mean-frame subtracted (see text).

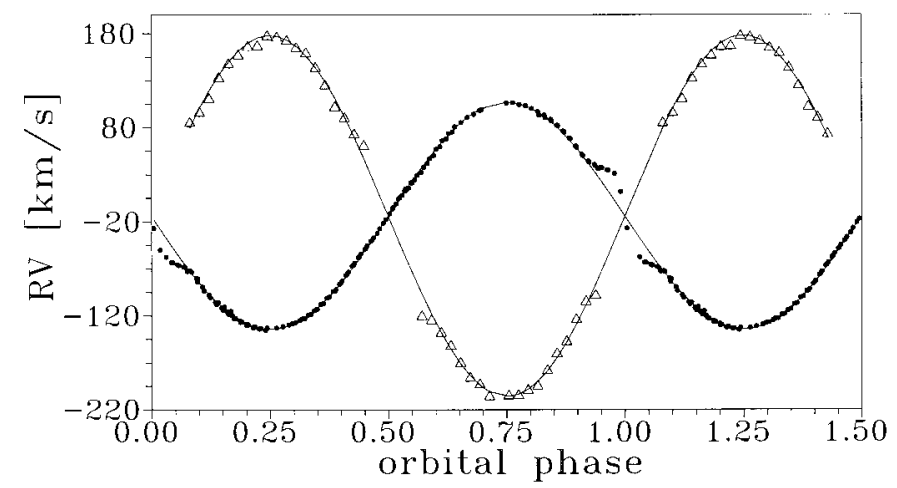

Fig. 3. Orbital solution for both components. $R V \mathrm{~s}$ obtained from the TLS spectra for the primary (dots) and for the secondary (triangles).

of SV Cam. We assume that the periodical variation observed in the $\mathrm{O}-\mathrm{C}$ values of the times of eclipses (for a compilation see e.g. ADD) are caused by light time effects due to a third body. We show that it is possible to calculate both the elements of the third body orbit and an optimized period of the eclipses themselves from the observed times of primary minima.

\subsection{The method}

Let $P_{0}$ be the period of the orbit of the primary around the center of mass with the secondary and $T_{0}$ the time of primary eclipse calculated regardless of the influence of a third body. All other elements now refer to the eclipsing-pair orbit around 
Table 4. Times of minimum light (-2400 000) used additionally to the data given by ADD. The Sonneberg data are, in a running order, from Mitteilungen der Sternwarte Sonneberg (Publications of the Sonneberg Observatory), Nos. 7, 32; 9, 80; 10, 42; 10, 42; 10, $104 ; 12,16$.

\begin{tabular}{lllllllll}
\hline \hline \multicolumn{2}{l}{ Sommer (1956) } & & & & & & & \\
13895.900 & 14850.737 & 15698.836 & 16499.475 & 17501.766 & 18498.118 & 19500.414 & 20496.766 & 21499.057 \\
22501.346 & 23497.701 & 24499.993 & 25549.727 & 25951.249 & 26799.333 & 26933.366 & 26946.410 & 26949.374 \\
26968.352 & 26980.217 & 27003.345 & 27134.412 & 27447.556 & 28132.566 & 28148.575 & 28196.616 & 28209.661 \\
28301.586 & 28563.725 & 28610.577 & 28862.635 & 29172.222 & 29174.592 & 29176.372 & 29177.564 & 29183.495 \\
29277.785 & 29286.681 & 29287.870 & 31745.566 & & & & & \\
33433.4483 & 33436.4077 & 33541.3807 & 33544.3488 & 33646.3680 & 33649.3319 & 33703.3003 & 33719.3096 & 33745.4067 \\
34121.4127 & 34134.4582 & 34169.4482 & 34201.4929 & 34439.2927 & 34452.3434 & 34455.3149 & 34605.3732 & 34780.3121 \\
34844.3661 & 35246.4701 & 35265.4523 & 35303.4034 & 35332.4678 & 35370.4208 & 35379.3225 & & \\
Braune et al. (1977) & 41764.319 & 42047.206 & 42404.242 & 42494.382 & & & \\
Sonneberg & 44559.398 & 44574.281 & 45281.225 & 45323.320 & 45651.303 & 47540.243 & \\
\hline
\end{tabular}

the common center of mass with the third body. For a Keplerian orbit, the O-C values vary according to (Mayer 1990)

$\mathrm{O}-\mathrm{C}=A\left[\eta^{2} \frac{\sin (\omega+v)}{1+e \cos v}+e \sin \omega\right]$

where

$A=\frac{K \eta P}{2 \pi c \sqrt{1-e^{2} \cos ^{2} \omega}}, \quad \eta=\sqrt{1-e^{2}}$.

Note that this equation only holds true for $T_{0}=T$, where $T$ is the time of periastron passage of the third body and $T_{0}$ is the time of primary eclipse. Otherwise a constant depending on $v\left(T_{0}-T\right)$ has to be added. Now we substitute the true anomaly $v$ by the eccentric anomaly $E$, related by

$\cos v=\frac{\cos E-e}{1-e \cos E}, \quad \sin v=\frac{\eta \sin E}{1-e \cos E}$

and expand Eq. (1) for $T_{0} \neq T$ by introducing the constant $C_{0}$ to get

$\mathrm{O}-\mathrm{C}=C_{0}+C_{1} \cos E+C_{2} \sin E$

where

$$
\begin{aligned}
& C_{1}=A \sin \omega, \quad C_{2}=A \eta \cos \omega \\
& C_{0}=-C_{1} \cos E\left(T_{0}\right)-C_{2} \sin E\left(T_{0}\right) .
\end{aligned}
$$

The time dependence of the eccentric anomaly $E$ is given by the Keplerian equation

$E(t)-e \sin E(t)=\frac{2 \pi}{P}(t-T)$

$E$ depends on the orbital period $P$ and on the orbital elements $e$ and $T$. These three parameters can be optimized so that the scatter of the residuals of a least squares fit according to Eq. (4) is at minimum. The other two elements can be directly calculated from the constants $C_{1}$ and $C_{2}$ of the optimum fit:

$K=\frac{2 \pi c}{\eta P} \sqrt{C_{1}^{2}+C_{2}^{2}}, \quad \tan \omega=\eta \frac{C_{1}}{C_{2}}$.

In this way we can determine the period and all elements of the third body orbit from a series of least squares fits searching for the optimum values in an $n$-dimensional parameter space.
The $\mathrm{O}-\mathrm{C}$ values itself are calculated from an adopted period of primary eclipses $P_{0}$. So, by varying $P_{0}$, we can optimize this period as well.

On the other hand, we can compute the $R V$ s directly from the measured $\mathrm{O}-\mathrm{C}$ values. The $R V$ variation of a Keplerian orbit is given by

$R V=K[e \cos \omega+\cos (\omega+v)]$

Substituting $v$ by $E$ we get

$R V=K \eta \frac{\eta \cos \omega \cos E-\sin \omega \sin E}{1-e \cos E}$.

By solving Eq. (4) for the eccentric anomaly $E$ and by inserting $E$ into Eq. (8) we get $R V$ as a function of $\mathrm{O}-\mathrm{C}$. The orbital elements can then be determined from the computed $R V \mathrm{~s}$ by usual orbital solutions using e.g. the method of differential corrections.

\subsection{Application and results}

We use the photoelectrically determined times of minimum light also used by ADD and supplement them by the early data by Sommer (1956) as well as by some newer data found in the literature (see Table 4).

Figure 5 shows the $\mathrm{O}-\mathrm{C}$ values calculated for three periods of light minima. The first period of 0.593071 given by Pojmanski (1998) was also used by ADD. The second period of 0.5930718 was given by Patkòs (1982) and the third of 0.5930720 is our spectroscopically obtained period. If we assume that the variation of the residuals is caused by the light time effect due to a third body we would expect a more or less sinusoidal variation without any long-term rising or falling trend. So in this respect the first period used by ADD is incompatible with the observed times of minimum light. The other two periods yield an $\mathrm{O}-\mathrm{C}$ variation as expected, the plot based on the 0.5930718 period shows the most adapted behaviour.

Figure 5 also shows that the photoelectric data used by ADD cover only sligthly more than one period whereas all data cover about two periods of variation. This was the reason for us to insert also the older data of lower accuracy into our investigation. 

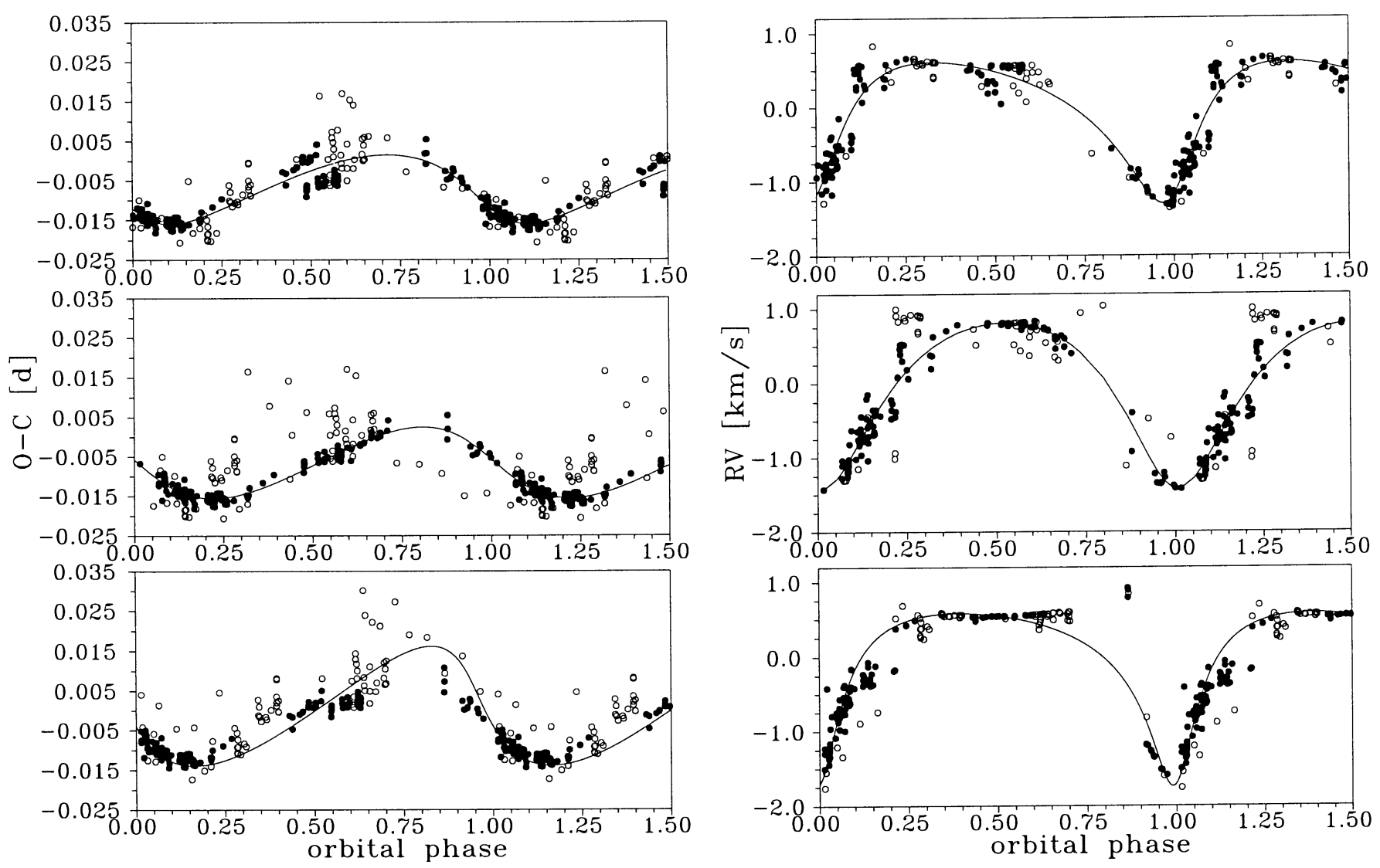

Fig. 4. Solutions for the third body orbit. The data set used by ADD is marked by filled circles. Left: best fit of O-C values. Right: calculated $R V \mathrm{~s}$ and the corresponding orbital curve. Note that $R V \mathrm{~s}$ could not be calculated for all of the observed $\mathrm{O}-\mathrm{C}$ values (see text). Top: obtained from all data and an optimized eclipse period of 0.5930718 , folded with the resulting orbital period of 52.3 yr. Center: obtained from the ADD data only and a fixed eclipse period of 0.5930718 , folded with the resulting orbital period of $43.5 \mathrm{yr}$. Bottom: obtained from all data, eclipse period fixed to the spectroscopic period of 0.5930720 , folded with the resulting orbital period of $55.3 \mathrm{yr}$.

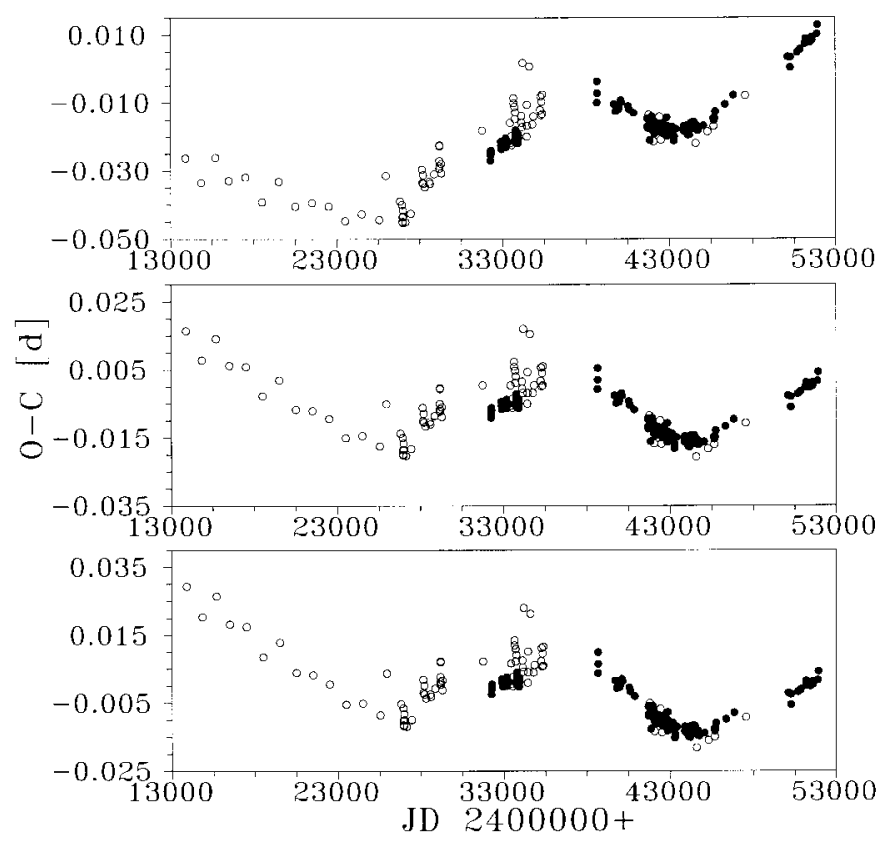

Fig. 5. O-C values calculated from the epochs of primary minima assuming a period of 0.593071 (top), of 0.5930718 (middle), and of 0.5930720 (bottom). The data also used by ADD are marked by filled circles.
The conversion of the observed $\mathrm{O}-\mathrm{C}$ values into $R V \mathrm{~s}$ mentioned as the second method in the previous section has the disadvantage that it can be done only for $|(\mathrm{O}-\mathrm{C})|<\left|C_{0}+\sqrt{C_{1}^{2}+C_{2}^{2}}\right|$, i.e. only for measured O-C values whose absolute amounts do not exceed the maxima of the curve given by Eq. (4). For that reason we prefer to use the first method of least squares fits and use the restricted conversion into $R V$ s only for a visualization of the results by phase diagrams showing $R V$ versus orbital phase. From the optimization of all parameters we get the results listed in the last row of Table 5. For comparison we list also the results by ADD, our fit to the ADD data, and the fit based on the period of the spectroscopic orbit. Figure 4 shows the corresponding phase diagrams. All results will be discussed in detail in Sect. 7.

\section{Stellar masses and radii}

The masses of the primary and secondary stellar components can be determined because the inclination angle is known to be very near to $90^{\circ}$. Assuming a circular orbit yields

$$
\begin{aligned}
& M_{1}=1.038 \times 10^{-7}\left(K_{1}+K_{2}\right)^{2} K_{2} P_{0}=1.09 \pm 0.05 M_{\odot} \\
& M_{2}=1.038 \times 10^{-7}\left(K_{1}+K_{2}\right)^{2} K_{1} P_{0}=0.70 \pm 0.02 M_{\odot} .
\end{aligned}
$$


From an analysis of the shape of the light-curve of SV Cam, Patkòs \& Hempelmann (1994) derived the radius of the primary in units of the semi-major axis of the relative orbit $a_{12}$ to $R_{1}=(0.3276 \pm 0.0015) a_{12}$ and the ratio of the radii of both components to $R_{2} / R_{1}=0.6431 \pm 0.0033$. Since we now know the value of $a_{12}$, it is

$a_{12}=\frac{86400}{2 \pi}\left(K_{1}+K_{2}\right) P_{0}=3.60 \pm 0.05 R_{\odot}$

we can calculate the absolute values of the stellar radii to get $R_{1}=1.18 \pm 0.02 R_{\odot}$ and $R_{2}=0.76 \pm 0.02 R_{\odot}$.

The motion of the center of masses of the primary and secondary component with respect to the third body with $K=$ $0.95 \mathrm{~km} \mathrm{~s}^{-1}, P=19103 \mathrm{~d}$ and $e=0.42$ results in a projected semi-major axis of the third body orbit of

$a_{3} \sin i=\frac{86400}{2 \pi} K P \sqrt{1-e^{2}}=2.265 \times 10^{8} \mathrm{~km}$

which gives a mass function of

$f(m)=\frac{M_{3}^{3} \sin ^{3} i}{\left(m+M_{3}\right)^{2}}=4 \times 10^{-20} \frac{\left(a_{3} \sin i\right)^{3}}{P_{3}^{2}}=0.0013 M_{\odot}$

where $m=M_{1}+M_{2}$ is the total mass of the two main components. In Table 6 we give the mass of the third body as a function of the unknown inclination angle.

\section{Discussion}

Spectroscopic orbit. The small time base of the high accuracy TLS data does not allow us to calculate an orbital period of sufficient accuracy to link with the early $R V \mathrm{~s}$ from the literature. So we supplemented our own data by the older low-accuracy $R V \mathrm{~s}$ to derive the orbital elements. Because of the strong weighting scheme, the accuracy of the derived $\gamma$-velocity, half-amplitude, and eccentricity is determined mainly by the TLS data whereas the very high precision of the orbital period is based on the entire data set spanning a time interval of more than half a century. For circular orbits we get a very precise epoch of primary conjunction (error of $15 \mathrm{~s}$ ).

If we allow for non-circularity, the derived eclipse epoch is of much lower accuracy because the very low eccentricity gives rise to large uncertainties in the epoch of periastron passage and in the longitude of periastron. Although the obtained eccentricity is significantly non-zero regarding its computed error there is some doubt on non-circularity. The reason is that we expect deviations from the orbital curve due to surface spots and possibly due to illumination effects which can certainly mimic a non-circular orbit of such low eccentricity.

Primary orbit from the times of light minima. From the $\mathrm{O}-\mathrm{C}$ values of published times of eclipses of SV Cam we calculate the orbital elements of a third body assuming that the observed periodic changes in the $\mathrm{O}-\mathrm{C}$ values are due to an additional orbital motion. For this purpose we derived an algorithm which is capable of calculating the period of light minima, independent of the spectroscopic orbit. As a result we get an orbital period of 0.5930718 (Table 6) different from the
Table 5. Orbital elements of the third body. We list the results of ADD, our best fit of the ADD data, the best fit of all data based on an optimized eclipse period, and the best fit of all data based on the period of the spectroscopic orbit. $T_{3}$ is the time of periastron passage in JD 2420000 . rms gives the mean scatter of the residuals. Errors of the last digit are given in parentheses.

\begin{tabular}{lrrrr}
\hline \hline & ADD & $\begin{array}{r}\text { ADD data } \\
P_{0} \text { fixed }\end{array}$ & $\begin{array}{r}\text { all data } \\
P_{0} \text { fixed }\end{array}$ & $\begin{array}{r}\text { all data, } P_{0} \\
\text { optimized }\end{array}$ \\
\hline$P_{0}[\mathrm{~d}]$ & .593071 & .5930718 & .5930720 & $\mathbf{. 5 9 3 0 7 1 8}$ \\
$P_{3}[\mathrm{yr}]$ & $41.3(3)$ & $44(1)$ & $55.3(5)$ & $\mathbf{5 2 . 3 ( 5 )}$ \\
$K\left[\mathrm{~km} \mathrm{~s}^{-1}\right]$ & & $1.12(3)$ & 1.16 & $\mathbf{0 . 9 5}(\mathbf{2})$ \\
$T_{3}$ & & $4700(700)$ & $1200(160)$ & $\mathbf{2 9 0 0 ( 4 0 0 )}$ \\
$e$ & $.29(3)$ & $.28(3)$ & $.54(2)$ & $\mathbf{. 4 2 ( 2 )}$ \\
$\omega\left[{ }^{\circ}\right]$ & $183(2)$ & $175(7)$ & $194(4)$ & $\mathbf{2 1 0}(\mathbf{4})$ \\
\hline $\mathrm{rms}_{\mathrm{OC}}$ & {$[\mathrm{d}]$} & & .0043 & $\mathbf{. 0 0 3 8}$ \\
$\mathrm{rms}_{\mathrm{RV}}$ & {$\left[\mathrm{km} \mathrm{s}^{-1}\right]$} & & .21 & $\mathbf{. 2 0}$ \\
\hline
\end{tabular}

Table 6. The mass of the third body as a function of orbital inclination.

\begin{tabular}{lrrrrrrrrr}
\hline \hline$i_{3}$ in $^{\circ}$ & 10 & 20 & 30 & 40 & 50 & 60 & 70 & 80 & 90 \\
$M_{3} / M_{\odot}$ & 1.35 & .56 & .37 & .28 & .23 & .20 & .18 & .17 & .17 \\
\hline
\end{tabular}

spectroscopic period of 0.5930720 (Table 3). Since we cannot give an error of the period derived from the eclipse times we checked for the compatibility of both values.

First we fixed the period to the spectroscopic period of 0.5930720 and calculated the third body elements. Results show that the 0.5930720 period gives a larger rms of the residuals (Table 5). Moreover, in the lower right panel of Fig. 4 one can see clear outliers in $R V$ at an orbital phase of about 0.85 . These $R V \mathrm{~s}$ were calculated from photoelectrical $\mathrm{O}-\mathrm{C}$ values and their position in respect to the orbital curve is not compatible with the mean scatter of all other RVs of photoelectric origin. In a second test we fixed the orbital period to 0.5930718 and re-calculated all other spectroscopic elements. Here we observed that the rms of the residuals is of the same amount as for the 0.5930720 period and also the phase diagrams showed no visible difference. So we assume that the error derived for the spectroscopic period is underestimated: The reason may be that we derived the weights of the individual data sets during an iterative procedure with the aim to minimize the rms of the orbital residuals. The errors of the orbital elements are minimized in this way as well regardless of the fact that we do not know the true data weights.

Finally, we adopt the period of 0.5930718 following from the times of primary eclipse as the most reliable orbital period. The same period length was also derived by Patkòs (1982) from the light-curves of SV Cam. Table 7 summarizes the estimated stellar parameters and the final orbital elements where the spectroscopic orbit was re-calculated by fixing the orbital period to 0.5930718 . The accurate, light time effect corrected times of primary eclipses can be calculated from

$\mathrm{JD}_{\text {p.e. }}=t+C_{1} \cos E(t)+C_{2} \sin E(t), \quad t=T_{0}+n P$ 
Table 7. Derived stellar parameters, final orbital elements, and parameters for the calculation of eclipse times according to Eq. (14). $T_{0}$ refers to the epoch of primary conjunction and $T_{3}$ to the epoch of periastron passage. Errors of the last digit are given in parentheses.

\begin{tabular}{|c|c|c|c|c|}
\hline \multicolumn{5}{|c|}{ primary/secondary } \\
\hline$M_{1}$ & $1.09(5) M_{\odot}$ & $P$ & 0.5930718 & $\mathrm{~d}$ \\
\hline$R_{1}$ & $1.18(2) R_{\odot}$ & $\gamma$ & $-13.9(2)$ & $\mathrm{km} \mathrm{s}^{-1}$ \\
\hline$M_{2}$ & $0.70(2) M_{\odot}$ & $K_{1} 1$ & $-120.5(2)$ & $\mathrm{km} \mathrm{s}^{-1}$ \\
\hline$R_{2}$ & $0.76(2) R_{\odot}$ & $K_{2}$ & $187(4)$ & $\mathrm{km} \mathrm{s}^{-1}$ \\
\hline \multicolumn{5}{|c|}{ third body } \\
\hline \multirow[t]{5}{*}{$M_{3}$} & $\geq 0.17 M_{\odot}$ & $P_{3}$ & $52.3(5)$ & $\mathrm{yr}$ \\
\hline & & $K_{3}$ & $0.95(2)$ & $\mathrm{km} \mathrm{s}^{-1}$ \\
\hline & & $T_{3}$ & $2422900(400)$ & \\
\hline & & $e_{3}$ & $0.42(2)$ & \\
\hline & & $\omega_{3}$ & $210(4)$ & $\circ$ \\
\hline \multicolumn{5}{|c|}{ calculation of eclipse times } \\
\hline & & $T_{0}$ & $2452011.4148(2)$ & \\
\hline & & $C_{1}$ & -0.00466 & \\
\hline & & $C_{2}$ & -0.00745 & \\
\hline
\end{tabular}

where the eccentric anomaly $E$ is given by

$E(t)-e \sin E(t)=\frac{2 \pi}{P_{3}}\left(t-T_{3}\right)$.

The values of $T_{0}, C_{1}$, and $C_{2}$ are listed in Table 7. $T_{0}$ is the epoch taken from the final orbital solution and corrected for the constant $C_{0}$ which was obtained from our parameter optimization according to Eq. (4), together with the constants $C_{1}$ and $C_{2}$.

Third body orbit. From Table 5 it can be seen that our third body orbital solution gives a longer period and a larger eccentricity compared to the results of ADD. If we use only the data set from the ADD study our method is not able to find an optimum period of the light minima. The reason is that the time basis of the data is too small and the best fit is found for a combination of the smallest period of light minima and the largest orbital period of the third body with very high orbital eccentricity. To check for the reason for the different orbital eccentricities we applied our procedure to the same data set as used by ADD but fixed the period to the value of 0.5930718 . The resulting phase diagrams (Fig. 4) show that the fit for the ADD data alone is very smooth. The older data from Sommer (1956) are not fitted at all, however. The obtained elements are very near to the results of ADD (Table 5). So the reason for the differences in orbital period and eccentricity is that we used an extended data set and not the use of a different period of light minima. Including the older data which cover about the same time basis one orbital cycle earlier gives a longer period of the third body orbit with larger eccentricity.

Mass of the third body. Because we know the inclination angle of the primary orbit we can directly deduce the masses of the primary and secondary star. For the third body we can only derive the mass function. Assuming that the third body orbit is most likely coplanar the most probable value of $M_{3}$ is $0.17 M_{\odot}$. Table 6 shows that a third body which is as massive as the primary or the secondary star requires an unlikely low orbital inclination. Moreover, when the third body is a main sequence star, masses of $\geq 0.6 M_{\odot}$ should be excluded because a third stellar spectrum would be visible in the observations which is not the case.

Rotational line broadening. From the radii derived in Sect. 6 and the orbital period length we get, assuming strictly bound rotation, a $v \sin i$ of $101 \mathrm{~km} \mathrm{~s}^{-1}$ for the primary and of $65 \mathrm{~km} \mathrm{~s}^{-1}$ for the secondary component. Our value for the primary is very close to the value of $105 \mathrm{~km} \mathrm{~s}^{-1}$ obtained by Pojmanski (1998) from spectral line analysis.

\section{Conclusions}

From the O-C values of the times of primary eclipse of SV Cam we derive an orbital period of 0.5930718. This period is the same as given by Patkòs (1982) and is compatible with the observed $R V$ variations of SV Cam. Adopting this period we calculate new spectroscopic orbital elements assuming a circular orbit. If we allow for non-circular orbits the resulting eccentricity is very low (0.01) and cannot be considered to be significant because of possible distortions of the $R V$ curve due to surface spots or illumination effects.

The derived masses of $M_{1}=1.09 \pm 0.05 M_{\odot}$ and $M_{2}=$ $0.70 \pm 0.02 M_{\odot}$ correspond to main sequence stars of spectral types G 0 and K 6, respectively (Schmidt-Kahler 1982). The stellar radii found to be $R_{1}=1.18 \pm 0.02 R_{\odot}$ and $R_{2}=0.76 \pm$ $0.02 R_{\odot}$ are very close to those which are expected from the ZAMS mass-radius relation (Schmidt-Kahler 1982) of $R_{1}=$ $1.09 \pm 0.04 R_{\odot}$ and $R_{2}=0.70 \pm 0.01 R_{\odot}$ (errors are calculated from the errors of the mass determination). The slight difference might be simply the result of an elliptical distortion of the stellar bodies which is clearly visible in the eclipsing light curve. Hence, our conclusion is that both components are not evolved but are main sequence stars.

From periodic changes of the $\mathrm{O}-\mathrm{C}$ values of the eclipse times we deduce new elements of a third body orbit. The resulting orbital period of 52 years is longer than the period obtained by ADD and the eccentricity of at least 0.42 is distinctly higher than the value of 0.29 given by ADD. The reason for the difference is that we used a larger data set spanning about two period cycles of the third body motion. A lower limit for the mass of the secondary can be estimated from the mass function. Assuming coplanarity, the most likely value of $M_{3}$ is $0.17 M_{\odot}$. We emphasize, however, that our analysis is based on the hypothesis of the existence of a third body. The final proof of this hypothesis can only be the detection of radial velocity variations due to the third body orbit. Unfortunately, all our $R V$ observations are placed on the flat portion of the orbital curves shown in Fig. 4 so that the expected $\gamma$-velocity variations are too small to be observable in our spectra.

In the residuals of the $R V$ s after subtracting the orbital solution (Fig. 1) one can clearly see variations of shorter time scale which are partly stable over more than one orbital cycle. The investigation of these short-term variations will be subject of a forthcoming paper, together with an investigation of the surface structure of SV Cam by Doppler imaging. 
Acknowledgements. The authors wish to thank Prof. A. Hatzes for his careful proofreading of the manuscript and the referee, Dr. A. G. Gunn, for useful hints which lead to a final improvement of the paper.

\section{References}

Albayrak, B., Demircan, O., Djurasevic, G., Erkapic, S., \& Ak, H. 2001, A\&A, 376, 158A (ADD)

Braune, W., Hübscher, J., \& Mundry, E. 1977, AN, 298, 121

Budding, E., \& Zeilik, M. 1987, ApJ, 319, 827

Busso, M., Scaltriti, F., \& Cellino, A. 1985, A\&A, 148, 29

Cellino, A., Scaltriti, F., \& Busso, M. 1985, A\&A, 144, 315

Frieboes-Conde, H., \& Herczeg, T. 1973, A\&AS, 12, 1

Hempelmann, A., \& Patkòs, L. 1991, AN, 312, 19

Hempelmann, A., Hatzes, A. P., Kuerster, M., \& Patkòs, L. 1997, A\&A, 317, 125

Hilditch, R. W., Harland, D. M., \& McLean, B. J. 1979, MNRAS, 187, 797

Hiltner, W. A. 1953, ApJ, 118, 262
Lanza, A. F., \& Rodonò, M. 1999, A\&A, 349, L887

Mayer, P. 1990, BAC, 41, 231

Moore, E., Minnaert, M. G. J., \& Houtgast, J. 1966, The solar spectrum $2935 \AA$ to $8770 \AA$, National Bureau of Standards Monograph 61 (Washington)

Özeren, F. F., Gunn, A. G., Doyle, J. G., \& Jevremović, D. 2001, A\&A, 366, 202

Patkòs, L. 1982, Contrib. Konkoly Obs., 80, 1

Patkòs, L., \& Hempelmann, A. 1994, A\&A, 292, 119

Pojmanski, G. 1998, Acta Astron., 48, 711

Rainger, P. P., Hilditch, R. W., \& Edwin, R. P. 1991, MNRAS, 248, 168

Schlesinger, F. 1908, Publ. Allegheny Obs., 1, 35

Schmidt-Kahler, T. H. 1982, in Landolt-Börnstein New Series, vol. 2b, ed. K. Schaifers, \& H. H. Voigt (Springer-Verlag N.Y. 1982)

Sommer, R. 1956, Astron. Nachr., 283, 155

Van Worden, H. 1957, Leiden Ann., 21, 3

Zeilik, M., DeBlasi, C., Rhodes, M., \& Budding, E. 1988, ApJ, 332, 293 